\title{
Salidroside attenuates oxidized low-density lipoprotein-induced endothelial cell injury via promotion of the AMPK/SIRT1 pathway
}

\author{
DONGMING ZHAO ${ }^{1}$, XINYI SUN ${ }^{2}$, SHUJIE LV ${ }^{1}$, MIYING SUN ${ }^{1}$, HUATAO GUO $^{1}$, YUJIA ZHAI ${ }^{1}$, \\ ZHI WANG ${ }^{1}$, PENG DAI $^{1}$, LINA ZHENG ${ }^{1}$, MINGZHE YE $^{1}$ and XINPENG WANG ${ }^{1}$ \\ Departments of ${ }^{1}$ Cardiovascular and ${ }^{2}$ Endocrinology and Metabolism, Affiliated Hospital of Beihua University, \\ Jilin, Jilin 132011, P.R. China
}

Received August 29, 2018; Accepted March 12, 2019

DOI: $10.3892 / \mathrm{ijmm} .2019 .4153$

\begin{abstract}
Oxidized low-density lipoprotein (ox-LDL)-induced endothelial damage contributes to the initiation and pathogenesis of atherosclerosis. Salidroside can alleviate atherosclerosis and attenuate endothelial cell injury induced by ox-LDL. However, the mechanisms involved in this process are not fully understood. Therefore, the purpose of the present study was to investigate the role of the adenosine monophosphate-activated protein kinase (AMPK)/sirtuin (SIRT) 1 pathway in the protection of salidroside against ox-LDL-induced human umbilical vein endothelial cells (HUVECs) injuries. The results revealed that salidroside reverses ox-LDL-induced HUVECs injury as demonstrated by the upregulation of cell viability and downregulation of LDH release. In addition, salidroside increased the expression of the SIRT1 protein in ox-LDL-treated HUVECs. Next, it was demonstrated that SIRT1 knockdown induced by transfection with small interfering (si)RNA targeting SIRT1 (siSRT1) abolished the protection of salidroside against ox-LDL-induced HUVECs injuries. This was illustrated by a decrease in cell viability and an increase in LDH release, caspase-3 activity and apoptosis rate. Furthermore, salidroside mitigated ox-LDL-induced reactive oxygen species production, upregulated malondialdehyde content and NADPH oxidase 2 expression and decreased superoxide dismutase and glutathione peroxidase activities, while these effects were also reversed by siSIRT1 transfection. In addition, it was demonstrated that salidroside suppressed ox-LDL-induced mitochondrial dysfunction as demonstrated by the increase in mitochondrial membrane potential and decreases in cytochrome $c$ expression, and $\mathrm{Bax} / \mathrm{Bcl}-2$ reductions. However, these effects were eliminated
\end{abstract}

Correspondence to: Dr Xinyi Sun, Department of Endocrinology and Metabolism, Affiliated Hospital of Beihua University, 12 Jiefang Middle Road, Jilin, Jilin 132011, P.R. China

E-mail: sunxiny0103@qq.com

Key words: salidroside, atherosclerosis, adenosine monophosphateactivated protein kinase/sirtuin 1 pathway, oxidative stress, mitochondrial dysfunction by SIRT1 knockdown. Finally, it was demonstrated that salidroside significantly upregulated the phosphorylated-AMPK expression in ox-LDL-treated HUVECs and AMPK knockdown induced by transfection with AMPK siRNA (siAMPK) leads to elimination of the salidroside-induced increase in cell viability and the decrease in LDH release. Notably, siAMPK transfection further decreased the expression of SIRT1. In conclusion, these results suggested that salidroside protects HUVECs against ox-LDL injury through inhibiting oxidative stress and improving mitochondrial dysfunction, which were dependent on activating the AMPK/SIRT1 pathway.

\section{Introduction}

Atherosclerosis, resulting in lipid accumulation, extracellular matrix protein deposition and calcification of the arterial intima and media, causes arterial stiffness and reduces elasticity (1). Atherosclerosis is a progressive pathological process that involves vascular endothelial injury, lipid infiltration, vascular smooth muscle cell proliferation, inflammatory immune response and oxidative stress, eventually leading to a series of coronary artery diseases including ischemic stroke, heart attack, and peripheral vascular disease which remain a predominant cause of morbidity and morbidity in aged people worldwide (1). Although atherosclerosis is a multifactorial disease, vascular endothelial cell injury is a driving force in its initiation and pathogenesis (2). Oxidized low-density lipoprotein (Ox-LDL), a particularly important risk factor in the development of atherosclerosis, has been reported to induce vascular endothelial dysfunction via triggering accumulation of abundant lipids in arterial walls, endothelial cell apoptosis, oxidative stress, mitochondrial dysfunction and permeability (3). Up to date, control of the development of atherosclerosis remains a great challenge due to the incomplete understanding of the involved molecular signaling pathways (4). Therefore, mitigating ox-LDL-induced endothelial cell injury and further elucidating the underlying mechanism may be potential therapeutic strategies for atherosclerosis prevention and therapy.

Oxidative stress has been recognized as one of the causative factors in atherosclerosis and reactive oxygen species (ROS) are considered to be signaling molecules that lead to endothelial dysfunction in clinical and experimental 
atherosclerosis (5). The mitochondrion is one of the main sources of chronic ROS production under physiological conditions (6). Mitochondrial dysfunction is conducive to the process of atherosclerosis as evidenced by human and animal models of oxidative stress (7). Oxidative stress and mitochondrial dysfunction are closely linked, giving rise to a vicious cycle of endothelial cell damage leading to the development of atherosclerosis (8-10). Therefore, therapeutic approaches involving inhibiting oxidation and improving mitochondrial function may prevent atherosclerosis progression.

Salidroside is an active component isolated from Rhodiola rosea, a well-known herb in traditional Chinese medicine, exerts various pharmacological properties including anti-inflammation (11), anti-apoptosis (12), antioxidative stress (13) and anti-endothelial dysfunction (14). In recent years, the beneficent effects of salidroside on atherosclerosis have attracted attention $(15,16)$. The study from Xing et al (17) revealed that salidroside has been demonstrated to attenuate endothelial cellular senescence through decreasing the inflammatory response in an atherosclerosis model. In addition, salidroside can protect against ox-LDL-induced foam cell formation and apoptosis in THP1 human acute monocytic leukemia cells (12). Although a number of studies have reported on the anti-atherosclerotic effect of salidroside, the molecular mechanisms underlying this effect are not well understood.

SIRT1, a member of the conserved sirtuin family is a key regulator in the progression of atherosclerosis, regulates a well-known survival mechanism in atherosclerosis (18). Studies have demonstrated that endothelial SIRT1 serves a protective role in the development of atherosclerosis through a variety of mechanisms including reducing apoptosis, improving endothelial function and defending against oxidative stress $(19,20)$. SIRT1 deficiency in endothelial cells contributes to promoting the process of atherosclerosis $(21,22)$. Adenosine monophosphate-activated protein kinase (AMPK), a fuel-sensing enzyme, takes part in the anti-atherosclerotic process through improving endothelial function in cardiovascular disease $(23,24)$. Notably, it is reported that AMPK can transcriptionally activate nicotinamide phosphoribosyl transferase and then induce the activation of SIRT1 (25). Growing evidence has demonstrated that the AMPK/SIRT1 pathway serves pivotal roles in anti-atherosclerosis pathogenesis. However, the role of the AMPK/SIRT1 pathway in the cardioprotective action of salidroside against atherosclerosis has not been determined and remains to be investigated.

Therefore, the present study investigated the protective effect of salidroside on ox-LDL-induced human umbilical vein endothelial cells (HUVECs) injury and focused on the roles of the AMPK/SIRT1 pathway in these processes. A successful study may provide a potential strategy for atherosclerosis therapy.

\section{Materials and methods}

Reagents and antibodies. Salidroside (cat. no. 43866-25, Purity $>98 \%$ ), ox-LDL (cat. no. BP368) and dichloro-dihydro-fluorescein diacetate (DCFH-DA; cat. no. D6883) were obtained from Sigma-Aldrich (Merck KGaA, Darmstadt, Germany). Salidroside and ox-LDL were diluted with DMSO and PBS, respectively, and stored at $-20^{\circ} \mathrm{C}$. Dulbecco's modified Eagle's medium (DMEM; cat. no. 10569010), fetal bovine serum (FBS; no. 10099-141), and streptomycin/penicillin (cat. no. 15140122) were purchased from Gibco (Thermo Fisher Scientific, Inc., Waltham, MA, USA). Small interfering (si)RNA targeting SIRT1 (siSIRT1) and AMPK (siAMPK), and control siRNA (siControl) were obtained from Santa Cruz Biotechnology, Inc. (Dallas, TX, USA). The MTT assay (cat. no. C0009) and bicinchoninic acid (BCA) protein assay (cat. no. P0010) were purchased from the Beyotime Institute of Biotechnology (Beijing, China). The kits for the measurement of lactate dehydrogenase (LDH) release (cat. no. A020-2), methane dicarboxylic aldehyde (MDA) content (cat. no. A003-1), superoxide dismutase (SOD) activity (cat. no. A001-3) and glutathione peroxidase (GSH-Px) level (cat. no. A005) were purchased from Nanjing Jiancheng Bioengineering Institute (Nanjing, China). 5, 58, 6, 68-Tetraethylbenzimidazolcarbocyanine iodide (JC-1) was supplied by BioVision (Inc., Milpitas, CA, USA; cat. no. 4999-100). The caspase-3 colorimetric assay kit was supplied by Enzo Life Sciences Inc. (Farmingdale, NY, USA; cat. no. BML-AK703-0001). The Annexin V-Fluorescein Isothiocyanate (FITC)/Propidium Iodide (PI) Apoptosis Detection kit was obtained from BD Biosciences (Franklin, Lakes, NJ, USA; cat. no. 556547). Anti-SIRT1 (cat. no. ab104833) and anti-NADPH oxidase 2 (NOX2; cat. no. ab133303) antibodies were obtained from Abcam (Cambridge, UK). Anti-phosphorylated (p)-AMPK (cat. no. 2537), AMPK (cat. no. 2793), cytochrome $c$ (cat. no. 11940), Bax (cat. no. 774), Bcl-2 (cat. no. 15071) and GAPDH (cat. no. 2118) antibodies were obtained from Cell Signaling Technology, Inc., (Danvers, MA, USA).

Cell culture and treatment. HUVECs were from the American Type Culture Collection (Manassas, VA, USA; cat. no. CRL-1730) and cultured in DMEM with $10 \%(\mathrm{v} / \mathrm{v})$ FBS and $1 \%$ streptomycin/penicillin (v/v) in a humidified $95 \%$ air- $5 \% \mathrm{CO}_{2}$ incubator at $37^{\circ} \mathrm{C}$. For ox-LDL treatment experiments, HUVECs were grown to $70-80 \%$ confluence and then switched to different concentrations of ox-LDL (10, $25,50,100$ and $200 \mu \mathrm{g} / \mathrm{ml}$ ) for 12,24 , or $72 \mathrm{~h}$. For protection experiments, HUVECs were pretreated with salidroside $(0.1$, 1 and $10 \mu \mathrm{g} / \mathrm{ml}$ ) for $1 \mathrm{~h}$ and incubated continuously for $24 \mathrm{~h}$ with ox-LDL (100 $\mu \mathrm{g} / \mathrm{ml})$. For mechanism research studies, the cells were pre-transfected with siSIRT1, siAMPK and siControl, and then treated with salidroside $(1 \mu \mathrm{g} / \mathrm{ml})$ for $1 \mathrm{~h}$ followed by co-incubation with ox-LDL (100 $\mu \mathrm{g} / \mathrm{ml})$ for $24 \mathrm{~h}$.

Cell transfection. HUVECs at the density of $4 \times 10^{5}$ cells/well were seeded in a six-well plate and then transfected with siSIRT1 $(50 \mathrm{nM})$, siAMPK (100 nM) or siControl $(100 \mathrm{nM}$; Santa Cruz Biotechnology, Inc., Dallas, TX, USA) using Lipofectamine ${ }^{\circledR} 2000$ (Invitrogen; Thermo Fisher Scientific, Inc.) in accordance with the manufacturer's protocol. The siSIRT1 sequences were 5'-CCCUCAAAGUAAGACCAG UTT-3' (sense) and 5'-ACUGGUCUUACUUUGAGG GAA-3' (antisense). The siAMPK sequences were 5'-GGA CAGGGAAGCCUUAAAUTT-3' (sense) and 5'-AUUUAA GGCUUCCCUGUCCTT-3' (antisense). The negative control siRNA sequences were 5'-UUCUCCGAACGUGUCACG UTT-3' (sense) and 5'-ACGUGACACGUUCGGAGAATT-3' (antisense). Briefly, cells were grown to $70-90 \%$ confluence 
at the time of transfection. Lipofectamine ${ }^{\circledR} 2000(10 \mu \mathrm{l})$ was gently agitated and diluted in Opti-MEM ${ }^{\circledR}$ I Medium (cat. no. 31985-070; Invitrogen; Thermo Fisher Scientific, Inc.) without serum $(150 \mu \mathrm{l})$ in a separate vessel and the mixture was incubated for $5 \mathrm{~min}$ at room temperature. siRNA or control siControl $(150 \mathrm{pmol})$ was diluted into in Opti-MEM ${ }^{\circledR} \mathrm{I}$ Medium without serum $(150 \mu \mathrm{l})$. Subsequently, diluted DNA was added to diluted Lipofectamine ${ }^{\circledR} 2000$ Reagent (1:1 ratio). Following incubation for $5 \mathrm{~min}$ at room temperature, the siRNA-lipid complex $(250 \mu \mathrm{l})$ was added to each well containing cells and medium. Following transfection for $6 \mathrm{~h}$, the transfection mixture was replaced with fresh growth medium. Transfection efficiency was measured using western blot analysis. Subsequent experiments with transfected cells were performed following transfection for $24 \mathrm{~h}$.

HUVECs viability assay. The viability of HUVECs was determined by 3-(4,5-dimethylthiazol-2-yl)-2, 5-diphenyltetrazolium bromide (MTT) assay according to the manufacturer's protocol. Cells at a density of $1 \times 10^{4}$ cells/well were plated onto a 96-well culture plate and treated as discussed above. Following treatment for $24 \mathrm{~h}$, the MTT regent $(0.5 \mathrm{mg} / \mathrm{ml})$ was supplemented added and co-incubated for $3 \mathrm{~h}$ at $37^{\circ} \mathrm{C}$. Subsequently, DMSO $(150 \mu \mathrm{l})$ was added to each well to fully dissolve the crystal formazan. The optical density (OD) value at $450 \mathrm{~nm}$ was then measured using a microplate reader (Bio-Tek Instruments, Inc., Winooski, VT, USA). Each experiment was performed three times.

$L D H$ release assay. The release of $\mathrm{LDH}$ was determined using the LDH assay kit following the manufacturer's protocol. Briefly, cells were seeded in 96-well plates at a density of $1 \times 10^{4}$ cells/well and treated as discussion above for $24 \mathrm{~h}$ at $37^{\circ} \mathrm{C}$. Then the supernatant was collected. After centrifuging at $1,000 \mathrm{x}$ g for $10 \mathrm{~min}$ at room temperature, the supernatant $(20 \mu \mathrm{l})$ was mixed with 2,4-dinitrophenylhydrazine $(20 \mu \mathrm{l})$, transferred at $37^{\circ} \mathrm{C}$ for $15 \mathrm{~min}$ and then $\mathrm{NaOH}(0.4 \mathrm{M}, 250 \mu \mathrm{l})$ was added into the mixture and co-incubated for a further $15 \mathrm{~min}$ at $37^{\circ} \mathrm{C}$. The absorbance was detected at $450 \mathrm{~nm}$ using a microplate reader (Bio-Tek Instruments, Inc.).

Flow cytometric assay for cell apoptosis. HUVECs apoptosis was performed using an Annexin V-FITC/PI kit according to the manufacturer's protocol. Briefly, cells were cultured in 6-well plates at the density of $1 \times 10^{5}$ cells/well and treated as discussion above. After incubation for $24 \mathrm{~h}$, cells were harvested using $0.05 \%$ trypsin and washed three times with ice-cold PBS, and then re-suspended in $1 \mathrm{X}$ binding buffer (500 $\mu \mathrm{l})$ included in the kit followed by mixed and incubated with $5 \mu \mathrm{l}$ of Annexin V-FITC reagent and $5 \mu \mathrm{l}$ of PI at room temperature for $15 \mathrm{~min}$. Cell apoptosis was analyzed on a FACScan flow cytometer (BD Biosciences). The percentage of cells in quadrant $2(\mathrm{Q} 2)$ and $4(\mathrm{Q} 4)$ were quantitatively analyzed using FCS Express 3.0 software (De Novo Software, Glendale, CA, USA), generating the total percentage of apoptotic cells at both early and late apoptotic stage or apoptotic index. All experiments were performed in triplicate.

Measurement of intracellular ROS generation. The intracellular ROS production was determined by a redox-sensitive fluorescent probe dichloro-dihydro-fluorescein diacetate (DCFH-DA) according to the protocol. Briefly, following treatment for $24 \mathrm{~h}$, HUVECs were washed twice with ice-cold PBS and then incubated with DCFH-DA $(10 \mu \mathrm{M})$ for $20 \mathrm{~min}$ at $37^{\circ} \mathrm{C}$. After washing twice with PBS, the fluorescence values were detected by a F97 PRO fluorescence spectrophotometer (Shanghai Lengguang Technology Co., Ltd., Shanghai, China). In addition, following incubation with DCFH-DA $(10 \mu \mathrm{M})$ for $20 \mathrm{~min}$ at $37^{\circ} \mathrm{C}$, the sample was collected and centrifuged at $800 \mathrm{x} \mathrm{g}$ for $5 \mathrm{~min}$ at room temperature and washed twice with PBS, and then the fluorescence intensity was measured using FACScan flow cytometer at excitation and emission wavelengths of 485 and $528 \mathrm{~nm}$, respectively.

Measurements of intracellular MDA content, SOD and GSH-Px activities. The MDA content, SOD and GSH-Px activities in HUVECs were measured using colorimetric assay kits according to the manufacturer's protocols. The MDA level was measured using the thiobarbituric acid method with a maximum absorbance at $532 \mathrm{~nm}$. The SOD activity was based on the combination of xanthine and xanthine oxidase and the absorbance was recorded at a wavelength of $550 \mathrm{~nm}$. GSH-Px activity was determined using the enzyme-catalyzed reaction product (reduced glutathione) at a wavelength of $412 \mathrm{~nm}$.

Assessment of mitochondrial membrane potential (MMP). MMP was assessed using a cationic fluorescent indicator JC-1 which can selectively enter mitochondria and change color from red to green when MMP decreases. Briefly, HUVECs were washed twice with PBS and then incubated with JC-1 $(200 \mu \mathrm{M})$ for $20 \mathrm{~min}$ at $37^{\circ} \mathrm{C}$ followed by washing with PBS to remove excess JC-1. The fluorescence was observed by a F97 PRO fluorescence spectrophotometer (Shanghai Lengguang Technology Co., Ltd.) at an excitation wavelength of $529 \mathrm{~nm}$ and an emission wavelength of $590 \mathrm{~nm}$.

Assessment of caspase- 3 activity. The caspase- 3 activity was determined using caspase- 3 colorimetric assay kit carried out as described by the protocol provided by the manufacturer. The principle of the assay is that acetyl-Asp-Glu-Val-Asp p-nitroanilide (Ac-DEVD-pNA) could be catalyzed by caspase-3 to produce a yellow substance p-nitroaniline, which reflects caspase-3 activity. In brief, HUVECs were collected and lysed with lysis buffer (provided by the kit) for $30 \mathrm{~min}$ on ice followed by centrifuging at $16,000 \mathrm{xg}$ for $15 \mathrm{~min}$ at $4^{\circ} \mathrm{C}$. The supernatant was then incubated with Ac-DEVD-pNA at $37^{\circ} \mathrm{C}$ for $2 \mathrm{~h}$. Finally, the absorbance was detected at $405 \mathrm{~nm}$ and the relative caspase-3 activities were calculated as follows: Caspase- 3 activity $(\%)=\left(\mathrm{OD}_{\text {treatment }}-\mathrm{OD}_{\text {treatment blank }}\right) /\left(\mathrm{OD}_{\text {control }}-\mathrm{OD}_{\text {control blank }}\right) \times 100 \%$.

Western blot analysis. HUVECs were harvested and lysed in pre-cooled RIPA buffer (cat. no. P0013B; Beyotime Institute of Biotechnology) containing pheylmethanesulfonyl fluoride $(0.2 \mathrm{mM})$. The cell lysate was then centrifuged at $13,400 \mathrm{x} \mathrm{g}$ for $10 \mathrm{~min}$ at $4^{\circ} \mathrm{C}$ and the total protein concentration was determined by the BCA protein assay kit according to the protocol provided by the manufacturer. Equal amounts of protein $(30 \mu \mathrm{g})$ were separated by a $12 \%$ SDS-PAGE and then transferred to a polyvinylidene difluoride membrane. The membrane was blocked with TBS Tween-20 (TBST) 
buffer [20 mM Tris-HCl (pH 8.0)/150 mM NaCl/0.05\% Tween-20] containing 5\% non-fat dairy milk for $2 \mathrm{~h}$ at room temperature and then incubated overnight at $4^{\circ} \mathrm{C}$ with the following antibodies: Rabbit anti-SIRT1 (1:1,000), rabbit anti-phosphorylated AMPK $(1: 2,000)$, rabbit anti-AMPK $(1: 2,000)$, rabbit anti-Bax $(1: 1,000)$, rabbit anti-Bcl-2 $(1: 1,000)$, rabbit anti-NOX2 $(1: 1,000)$, rabbit anti-cytochrome $c(1: 1,000)$ or rabbit anti-GAPDH $(1: 1,000)$. After washing five times with TBST buffer, the membrane was incubated with horseradish peroxidase-conjugated goat anti-rabbit secondary antibody (1:5,000; cat. no. 7074; Cell Signaling Technology, Inc.) at for $2 \mathrm{~h}$ at room temperature. The membrane was finally visualized with the BeyoECL Plus detection kit (cat. no. P0018M; Beyotime Institute of Biotechnology). Scanned images were visualized with the LI-COR Odyssey Infrared Imaging System (LI-COR Biosciences, Lincoln, NE, USA).

Statistical analysis. Results are expressed as the mean \pm standard deviation from at least three separate experiments. Statistical analyses were performed using SPSS 13.0 software (SPSS, Inc., Chicago, IL, USA). The significance of differences was calculated with one-way analysis of variance followed by Tukey's test as appropriate. $\mathrm{P}<0.05$ was considered to indicate a statistically significant difference.

\section{Results}

Salidroside prevents ox-LDL-induced HUVECs injuries. To determine the cytotoxic potential of salidroside on HUVECs, the effects of salidroside on cell viability was evaluated. The MTT assay result demonstrated that incubation with low concentrations of salidroside $(0.1,1,5$, or $10 \mu \mathrm{M})$ for $24 \mathrm{~h}$ did not affect cell viability, whereas high concentrations of salidroside $(20,40$, or $80 \mu \mathrm{M})$ significantly reduced cell viability $(\mathrm{P}<0.05$; Fig. $1 \mathrm{~A})$. Therefore, three concentrations of salidroside $(0.1,1$ and $10 \mu \mathrm{M})$ were selected for subsequent experiments. Next, in order to investigate the damaging effects of ox-LDL on HUVECs, cells were treated with ox-LDL $(25,50,100$ and $200 \mu \mathrm{g} / \mathrm{ml})$ for 12,24 or $72 \mathrm{~h}$. The MTT assay results revealed that cell viability of HUVECs in response to ox-LDL (100 and $200 \mu \mathrm{g} / \mathrm{ml}$ ) for 12, 24 and $72 \mathrm{~h}$ was significantly reduced $(\mathrm{P}<0.05$; Fig. 1B). Notably, incubation with ox-LDL $(100 \mu \mathrm{g} / \mathrm{ml})$ for $24 \mathrm{~h}$ could reduce cell viability to $51.37 \pm 7.63 \%$, which was selected as the next experimental condition. Subsequently, it was demonstrated that pretreatment with salidroside $(0.1,1$, or $10 \mu \mathrm{M})$ significantly reversed ox-LDL-induced the downregulation of cell viability $(\mathrm{P}<0.05$; Fig. 1C). Furthermore, the LDH release assay result revealed that ox-LDL treatment significantly increased the LDH release in HUVECs $(\mathrm{P}<0.01)$, while this effect was also significantly inhibited by pretreatment with salidroside $(\mathrm{P}<0.05$; Fig. 1D). Flow cytometry with Annexin V-FITC/PI staining demonstrated that salidroside pretreatment significantly reversed the ox-LDL-induced upregulation of the apoptosis percentage $(\mathrm{P}<0.05$; Fig. $1 \mathrm{E}$ and $\mathrm{F})$. These results suggested that salidroside protects HUVECs from ox-LDL injury.

SIRT1 mediates the protection of salidroside from cytotoxicity and apoptosis induced by ox-LDL in HUVECs. SIRT1 serves a vital role in promoting cell survival in the development of atherosclerosis (26). In the present study, it was demonstrated that ox-LDL $(100 \mu \mathrm{g} / \mathrm{ml})$ treatment significantly reduced the expression of SIRT1, while this function was significantly abolished by pretreated with salidroside $(0.1,1$, or $10 \mu \mathrm{M}$; $\mathrm{P}<0.05$; Fig. 2A). According to the Fig. 1 and this result, it was demonstrated that salidroside $(10 \mu \mathrm{M})$ has a protective effect on ox-LDL injury and ox-LDL-induced SIRT expression downregulation. Therefore, salidroside $(10 \mu \mathrm{M})$ was selected for subsequent experiments. To further confirm the role of SIRT1 in the cardioprotection of salidroside, HUVECs were transfected with siSIRT1 or siControl. The transfection efficiency was detected using western blotting analysis and the result demonstrated that the expression of SIRT1 in siSIRT1 transfected cells was decreased compared with in the siControl transfected cells (Fig. 2B), indicating that SIRT1 knockdown was achieved by siSIRT1 transfection. In addition, it was demonstrated that ox-LDL-induced downregulation of cell viability was exacerbated by SIRT1 knockdown and the salidroside-induced increase in the cell viability was also significantly inhibited by siSIRT1 transfection in ox-LDL-treated HUEVCs (Fig. 2C). siSIRT1 transfection also further induced the increase in LDH release and reversed salidroside-induced the decrease in the LDH release in ox-LDL-treated HUEVCs (Fig. 2D). In addition, salidroside eliminated, while siSIRT1 exacerbated, ox-LDL-induced the upregulation of apoptosis ratio (Fig. 2E and F) and caspase-3 activity (Fig. 2G). Notably, the inhibition of salidroside on apoptosis was inhibition by transfection with siSIRT1. Together, the results suggested that SIRT1 contributes to the protection of salidroside against ox-LDL injury in HUVECs.

SIRT1 participates in the inhibitory action of salidroside against oxidative stress induced by ox-LDL in HUVECs. Oxidative stress is involved in the progression of atherosclerosis, which leads to ox-LDL-induced endothelial dysfunction (8). The effects of salidroside on the oxidative stress index including ROS generation, MDA content and NOX2 expression were further investigated with ox-LDL treatment. The result demonstrated that ox-LDL treatment results in an increase in ROS green fluorescence (Fig. 3A), DCF fluorescence percentage (Fig. 3B), MDA content (Fig. 3C) and NOX2 expression (Fig. 3D and E), which were reversed by salidroside. Notably, the effects of salidroside were reversed by SIRT1 knockdown induced by siSIRT1 transfection. Furthermore, siSIRT1 transfection further aggravated ox-LDL-induced oxidative stress. The antioxidants produced endogenously including SOD and GSH-Px serve important roles in preventing oxidative damage in atherosclerosis (27). It was demonstrated that salidroside diminished the ox-LDL-induced downregulation of SOD (Fig. 3F) and GSH-Px activities (Fig. 3G). However, the functions of salidroside were inhibited by siSIRT1 transfection. Taken together, these results indicated that SIRT1 mediates the protective effect of salidroside against oxidative stress and inhibition of antioxidant defense system induced by ox-LDL.

Salidroside attenuates ox-LDL-induced mitochondrial dysfunction via upregulation of SIRT1 in HUVECs. ROS produced by mitochondrial dysfunction affecting cell survival and resulting in apoptosis and oxidative stress have 
A

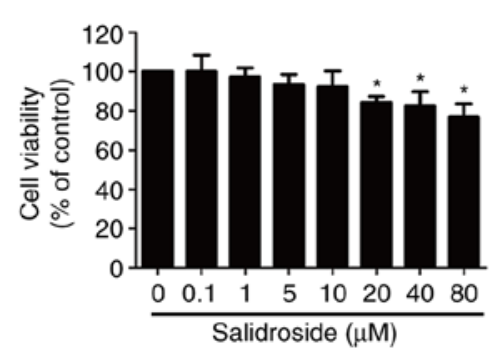

C

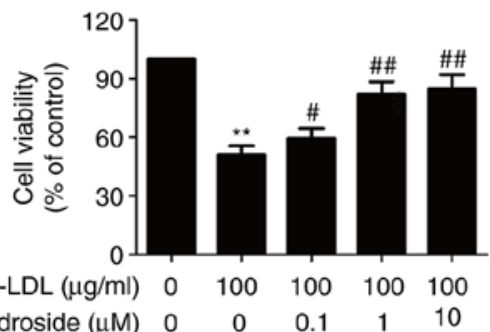

B

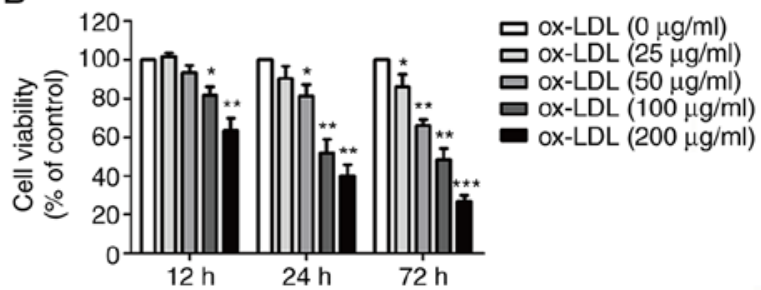

$\mathrm{D}$

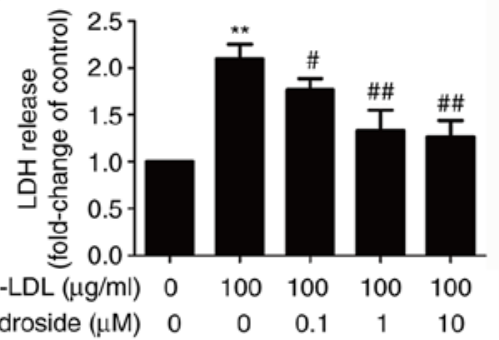

E
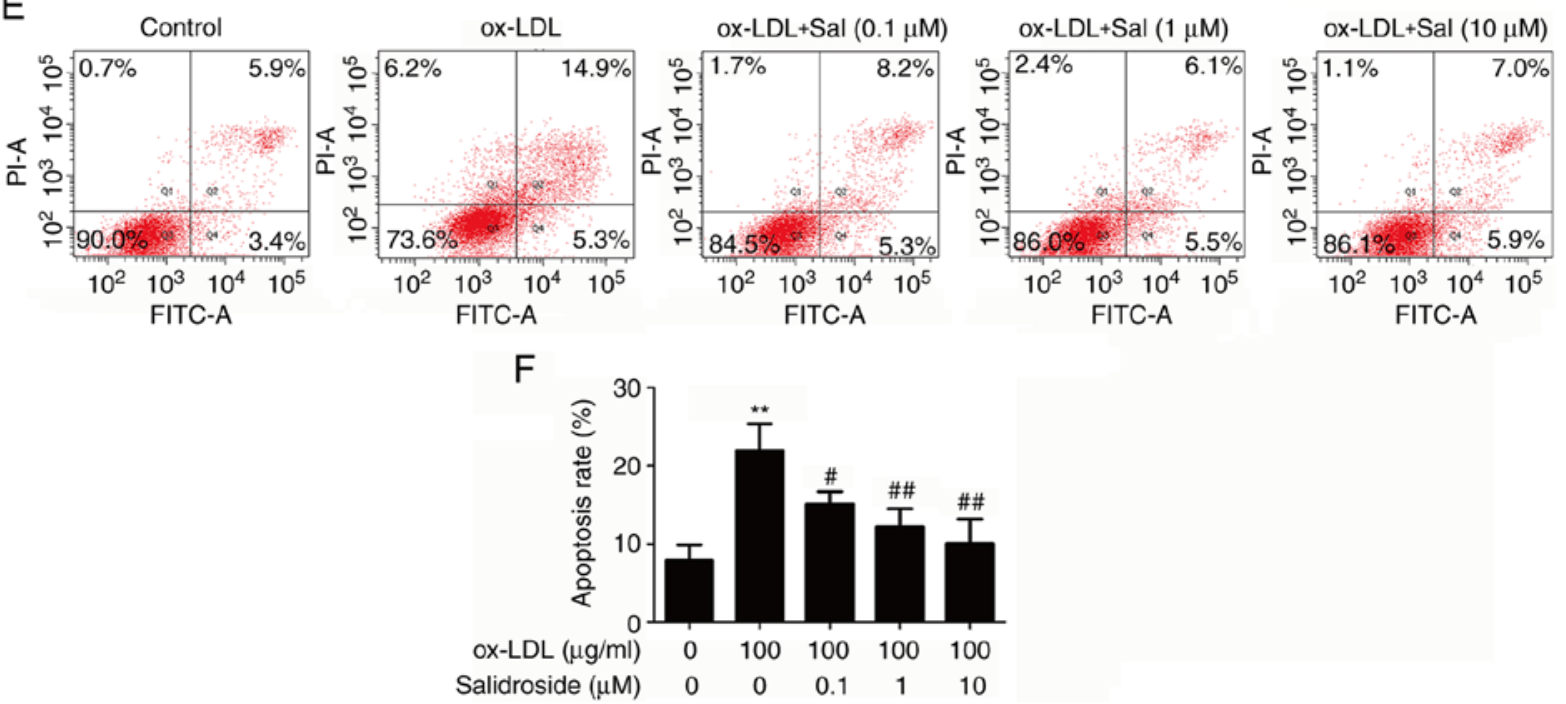

Figure 1. Effects of Sal on cytotoxicity in the presence or absence of ox-LDL in HUVECs. (A) HUVECs were incubated with Sal $(0.1,1,10,20,40$, or $80 \mu \mathrm{M})$ for $24 \mathrm{~h}$ and the cell viability was assessed by performing an MTT assay. (B) HUVECs were treated with different concentrations of ox-LDL (50, 100 and $200 \mu \mathrm{g} / \mathrm{ml})$ for $24 \mathrm{~h}$, and the cell viability was measured by MTT assay. HUVECs were pretreated with salidroside $(0.1,1$, or $10 \mu \mathrm{M})$ for $1 \mathrm{~h}$ and then exposed to ox-LDL $(100 \mu \mathrm{g} / \mathrm{ml})$ for $24 \mathrm{~h}$. (C) Cell viability was detected using an MTT assay. (D) LDH release was determined using an LDH assay kit. (E) The apoptosis ratio was measured using flow cytometry with an Annexin V-FITC/PI staining kit. (F) Quantitative analysis of Annexin V-FITC(+)/PI(-) and Annexin V-FITC $(+) / \mathrm{PI}(+)$. Data are expressed as the mean \pm standard deviation; $\mathrm{n}=3,{ }^{*} \mathrm{P}<0.05,{ }^{* *} \mathrm{P}<0.01$ and ${ }^{* * * *} \mathrm{P}=0.001$ vs. the control group; ${ }^{*} \mathrm{P}<0.05$ and ${ }^{\# \#} \mathrm{P}<0.01$ vs. the ox-LDL group. HUVECs, human umbilical vascular endothelial cells; ox-LDL, oxidized-low density lipoprotein; FITC, fluorescein isothiocyanate; PI, propidium iodide; LDH, lactate dehydrogenase; Sal, salidroside.

been implicated in the induction of atherosclerosis $(28,29)$. Therefore, whether salidroside effects mitochondrial function in ox-LDL-treated HUVECs and whether SIRT1 pathway is involved in this effect was investigated. JC-1 staining revealed that ox-LDL treatment resulted in the decrease in red fluorescence and the increase in green fluorescence indicating a lowering of MMP, while salidroside pretreatment resulted in the increase red fluorescence and the decrease green fluorescence, leading to increased MMP (Fig. 4A). However, the effect of salidroside was inhibited by siSIRT transfection. As an increase in cytochrome $c$ expression and $\mathrm{Bax} / \mathrm{Bcl}-2$ ratio are associated with MMP disruption and mitochondrial dysfunction (29), the effects of salidroside on these markers of mitochondrial function were analyzed. The results of western blot analyses (Fig. 4B) demonstrated that salidroside significantly reduced the expression of cytochrome $c(\mathrm{P}<0.05$; Fig. 4C) and the ratio of Bax/Bcl-2 compared with the ox-LDL treatment group, while these roles were abolished by transfection with siSIRT1 (Fig. 4D). These results suggested that salidroside improves mitochondrial dysfunction under ox-LDL injury conditions through promoting SIRT1 in HUVECs.

AMPK/SIRT1 pathway activation mediates the protective effect of salidroside against ox-LDL-induced HUVEC injury. AMPK is a critical regulator of mitochondrial biogenesis and is reported to enhance SIRT1 (30). To further certify the role of the AMPK pathway in cardioprotection by salidroside the effects of salidroside on the AMPK pathway following ox-LDL treatment were measured. It was demonstrated that ox-LDL decreased the $\mathrm{p}$-AMPK protein expression in 

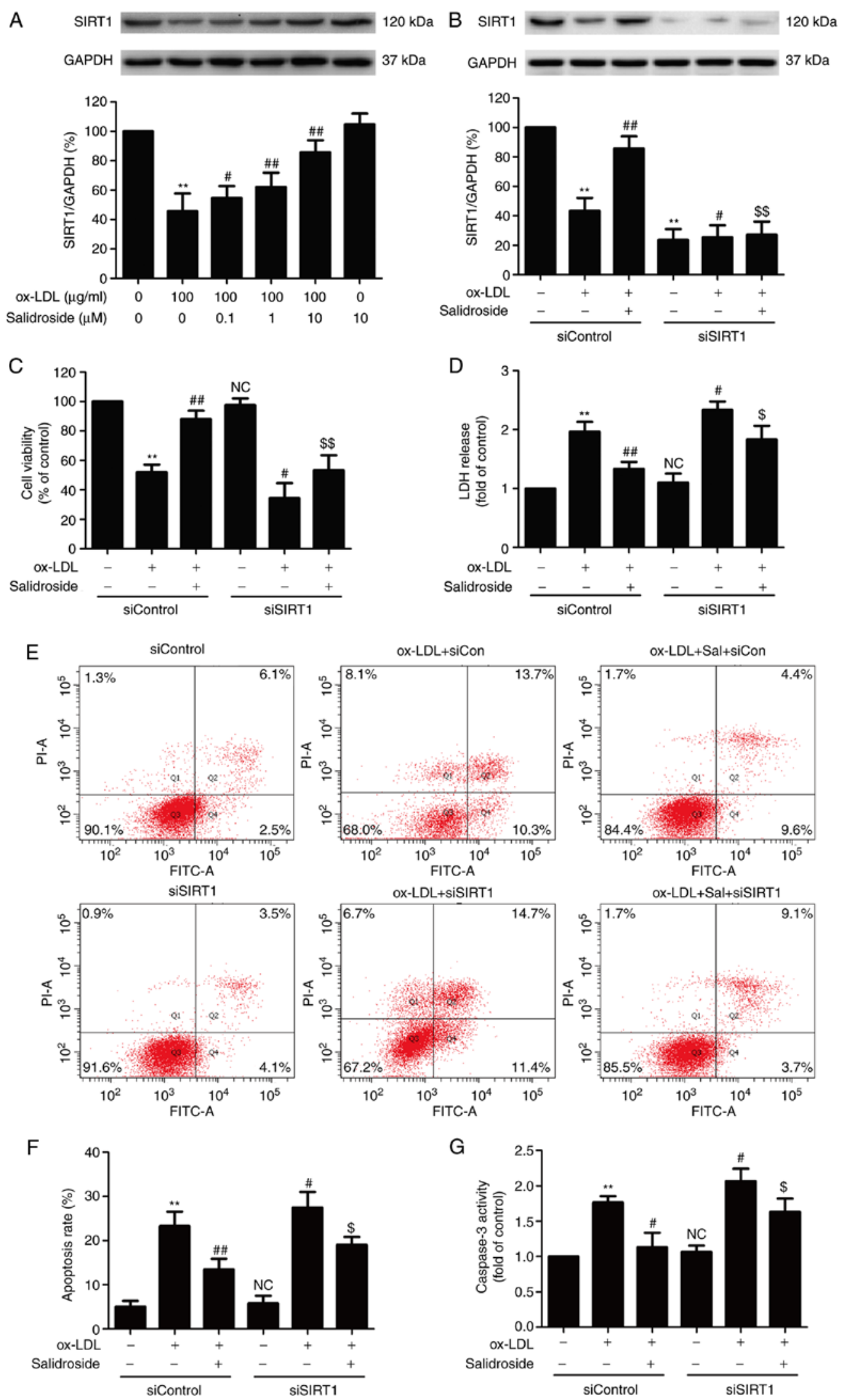

Figure 2. Effects of SIRT1 knockdown on the protection of Sal on ox-LDL-induced HUVECs injury. (A) Cells were pretreated with Sal $(0.1,1$, or $10 \mu \mathrm{M})$ for $1 \mathrm{~h}$ and then stimulated with ox-LDL $(100 \mu \mathrm{g} / \mathrm{ml})$ for $24 \mathrm{~h}$, and the expression of SIRT1 was measured by western blot analysis. HUVECs were pre-transfected with siSRT1 or siControl and incubated with Sal $(10 \mu \mathrm{M})$ for $1 \mathrm{~h}$ followed by treatment with ox-LDL $(100 \mu \mathrm{g} / \mathrm{ml})$ for $24 \mathrm{~h}$. (B) The transfection efficiency was determined by western blot analysis. (C) Cell viability was detected using MTT assay. (D) The LDH release was determined using an LDH assay kit. (E) The apoptosis was detected using the Annexin V-FITC/PI staining kit. (F) Quantitative analysis of Annexin V-FITC(+)/PI(-) and Annexin V-FITC(+)/PI(+). (G) The caspase-3 activity was measured by the caspase- 3 colorimetric assay kit. Data are expressed as the mean \pm standard deviation; $n=3$, ** $\mathrm{P}<0.01 \mathrm{vs}$. the control group; ${ }^{\#} \mathrm{P}<0.05$ and ${ }^{\# \#} \mathrm{P}<0.01$ vs. the ox-LDL group; ${ }^{\$} \mathrm{P}<0.05$ and ${ }^{\$ \$} \mathrm{P}<0.01$ vs. the ox-LDL and Sal co-treatment group. si, small interfering; HUVECs, human umbilical vascular endothelial cells; ox-LDL, oxidized low density lipoprotein; FITC, fluorescein isothiocyanate; PI, propidium iodide; LDH, lactate dehydrogenase; Sal, salidroside; NC, not significant; SIRT1, sirtuin 1. 

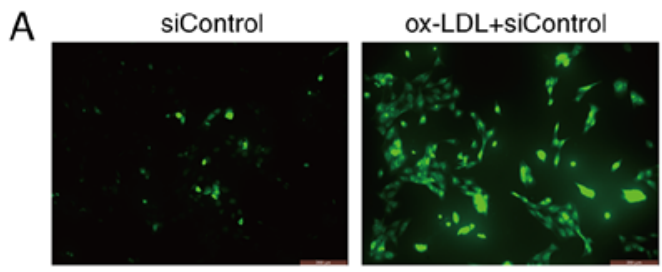

B

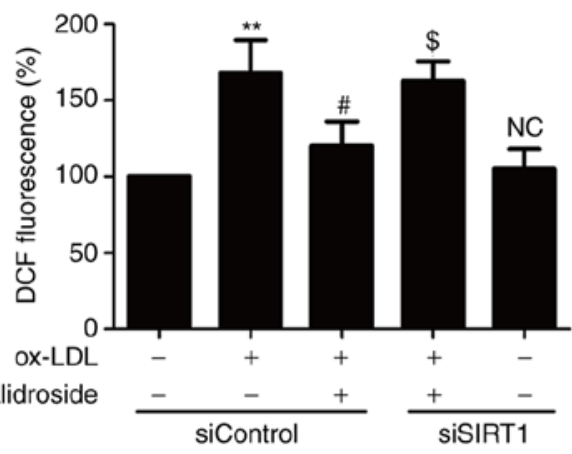

D
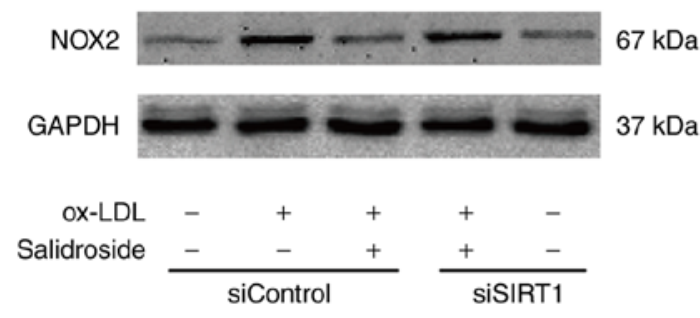

$\mathrm{F}$

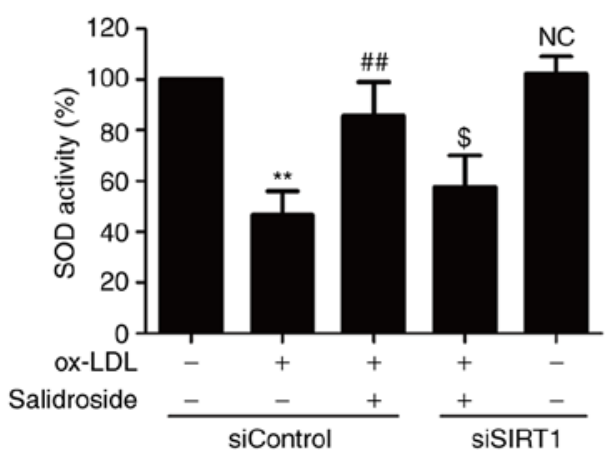

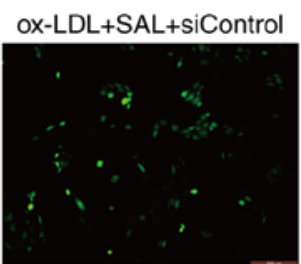
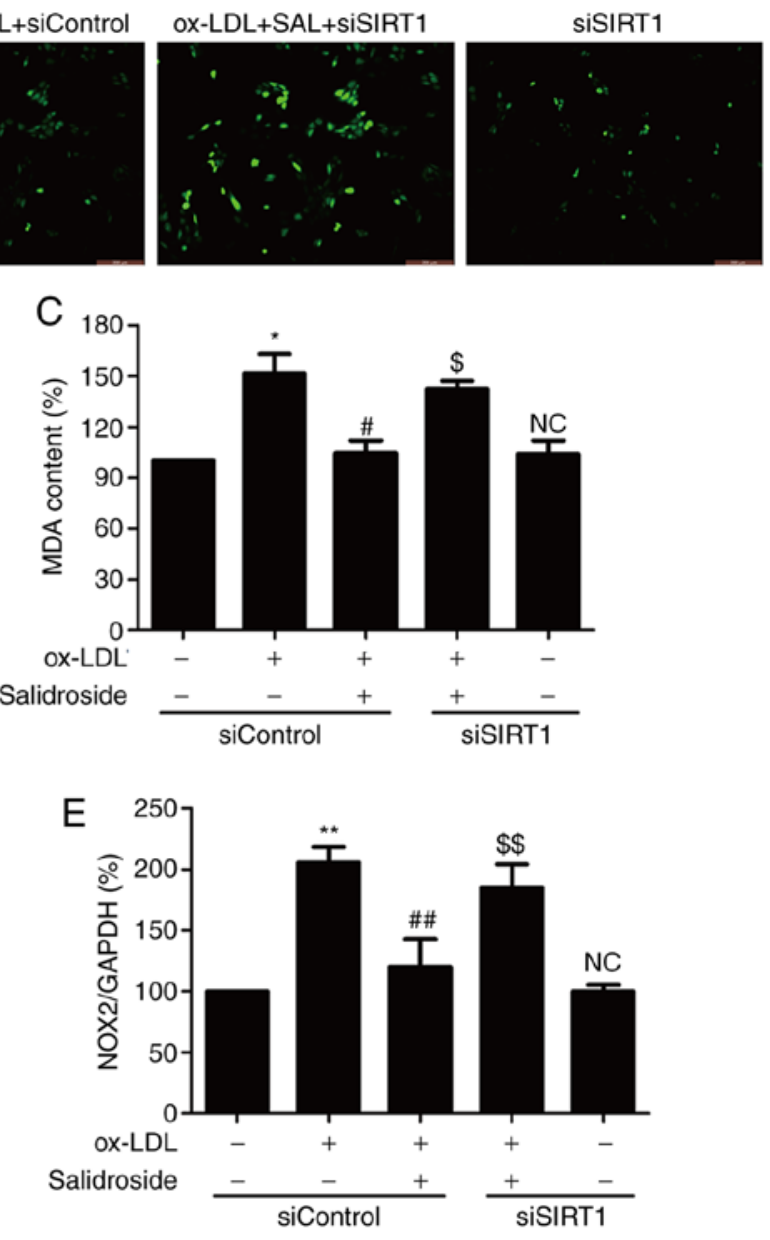

G

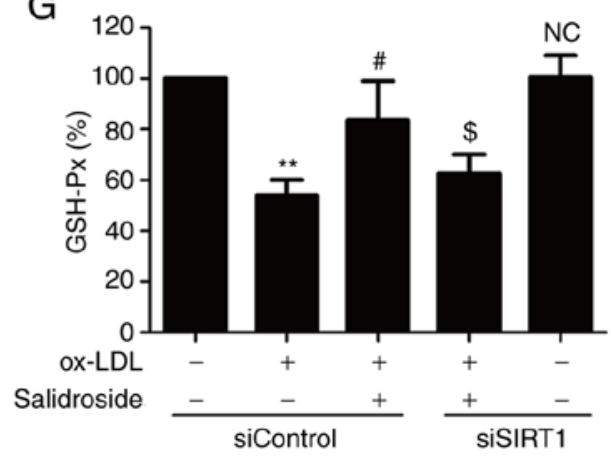

Figure 3. Effects of SIRT1 knockdown on the inhibition of salidroside on ox-LDL-induced oxidative stress in HUVECs. HUVECs were pre-transfected with siSRT1 or siControl and incubated with SAL $(10 \mu \mathrm{M})$ for $1 \mathrm{~h}$ followed by treatment with ox-LDL $(100 \mu \mathrm{g} / \mathrm{ml})$ for $24 \mathrm{~h}$. (A) The intracellular ROS production was determined by a redox-sensitive fluorescent probe DCFH-DA staining. Scale bar, $200 \mu \mathrm{M}$. (B) The fluorescence intensity of DCFDA-stained sample was quantified by flow cytometry. (C) The intracellular MDA content was measured using the thiobarbituric acid method with a maximum absorbance at $532 \mathrm{~nm}$. (D) The expression of NOX2 was determined using western blot analysis. (E) Bar charts demonstrate the quantification of NOX2 expression. (F) The SOD activity was detected using a colorimetric assay kit based on the combination of xanthine and xanthine oxidase. (G) The GSH-Px level was determined using a colorimetric assay kit depending on the enzyme-catalyzed reaction product (reduced glutathione). Data are expressed as the mean \pm standard deviation; $\mathrm{n}=3$, ${ }^{*} \mathrm{P}<0.05$ and ${ }^{* *} \mathrm{P}<0.01$ vs. the control group; ${ }^{\#} \mathrm{P}<0.05$ and ${ }^{\# \#} \mathrm{P}<0.01$ vs. the ox-LDL treatment group; ${ }^{\$} \mathrm{P}<0.05$ and ${ }^{\$ \$} \mathrm{P}<0.01$ vs. the ox-LDL and $\mathrm{SAL}$ co-treatment group. HUVECs, human umbilical vascular endothelial cells; ox-LDL, oxidized-low density lipoprotein; GSH-Px, glutathione peroxidase; SOD, superoxide dismutase; DCFH-DA, dichloro-dihydro-fluorescein diacetate; NOX2, NADPH oxidase 2; MDA, methane dicarboxylic aldehyde; ROS, reactive oxygen species; SAL, salidroside; NC, not significant; si, small interfering.

HUVECs, however salidroside significantly upregulated the p-AMPK expression in ox-LDL-treated HUVECs $(\mathrm{P}<0.05$; Fig. 5A and B), indicating that AMPK was activated by salidroside. To further investigate the roles of the AMPK pathway in the protective action of salidroside on ox-LDL injury, HUVECs were transfected with siAMPK. Western blotting analyses validation demonstrated that siAMPK transfection reduced the expression of AMPK compared with siControl transfection (Fig. 5C) following ox-LDL treatment or ox-LDL + salidroside co-treatment. In addition, it was demonstrated that transfection with siAMPK leads to the elimination of the salidroside-induced increase in cell viability (Fig. 5D) and the decrease in LDH release (Fig. 5E) in ox-LDL-treated HUVECs. Notably, it was demonstrated 
A
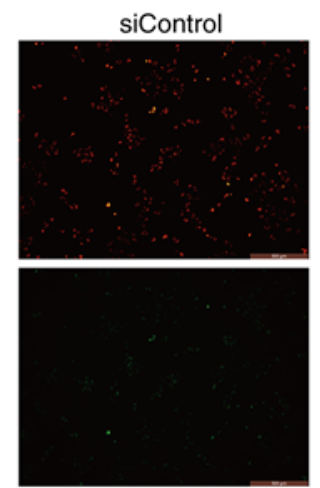

B

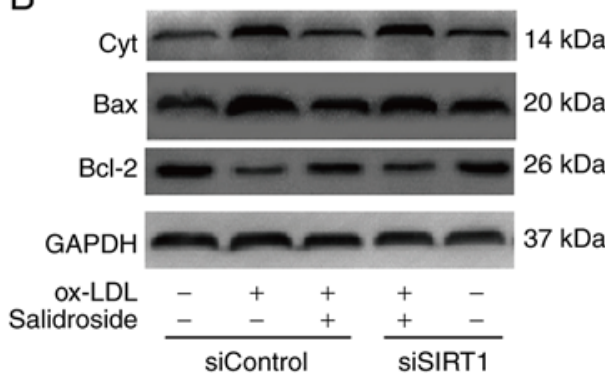

ox-LDL+siControl
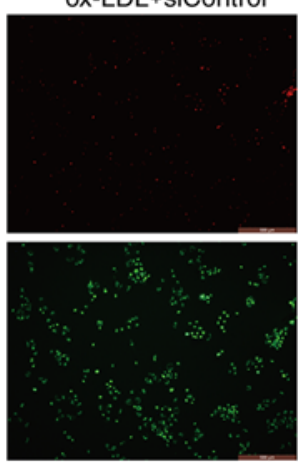

C

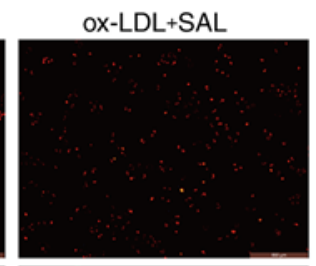

ox-LDL+SAL+siSIRT1
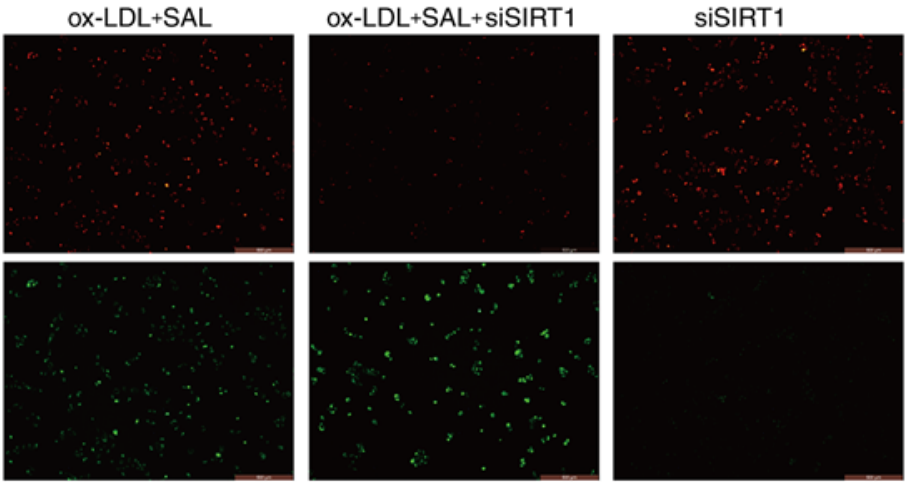

D

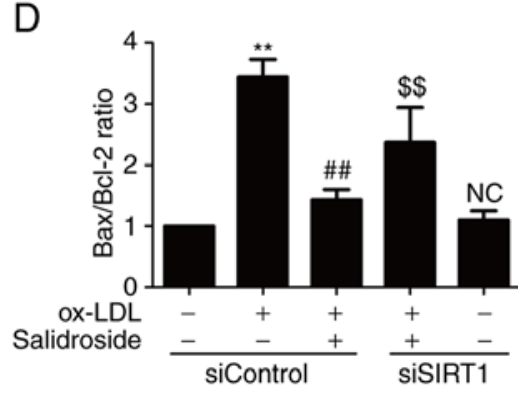

Figure 4. Effects of SIRT1 knockdown on the protection of salidroside-induced the improvement of mitochondrial dysfunction induced by ox-LDL in HUVECs. HUVECs were pre-transfected with siSIRT1 or siControl and incubated with salidroside $(10 \mu \mathrm{M})$ for $1 \mathrm{~h}$ followed by treatment with ox-LDL (100 $\mu \mathrm{g} / \mathrm{ml})$ for 24 h. (A) MMP was determined by the specific dye 5, 58, 6, 68-tetraethylbenzimidazolcarbocyanine iodide staining. Scale bar, $200 \mu \mathrm{M}$. (B) The expression of Cyt, Bax and Bcl-2 were measured by western blot analyses. Bar charts demonstrate the quantification of (C) Cyt and (D) the ratio of Bax/Bcl-2. Data are expressed as the mean \pm standard; $\mathrm{n}=3 .{ }^{* *} \mathrm{P}<0.01$ vs. the control group; ${ }^{\# \#} \mathrm{P}<0.01$ vs. the ox-LDL treatment group; ${ }^{\$} \mathrm{P}<0.05$ and ${ }^{\$ \$} \mathrm{P}<0.01$ vs. the ox-LDL and salidroside co-treatment group. NC, not significant; Cyt, cytochrome c; MMP, mitochondrial membrane potential; HUVECs, human umbilical vascular endothelial cells; ox-LDL, oxidized low density lipoprotein; si, small interfering; SIRT1, sirtuin 1.

that siAMPK transfection further decreased the expression of SIRT1 compared with the siControl transfection and also reversed salidroside-induced the upregulation of SIRT1 expression (Fig. 5F). In conclusion, the results of the present study suggested that salidroside protects HUVECs against ox-LDL-induced injury through activating the AMPK/SIRT1 pathway.

\section{Discussion}

Mounting evidence points to endothelial cell damage as one of the major pathophysiological links between cardiovascular risk factors and the development of atherosclerosis (31). Salidroside, a major active ingredient from the medicinal plant Rhodiola rosea L., possesses anti-inflammatory, anti-apoptotic antioxidative and cardioprotective effects (32-35). Although salidroside has been proven to be useful in treating various types of diseases including atherosclerosis, its effects on endothelial cell damage in atherosclerosis and the possible underlying molecular mechanisms remain unclear. In the present study, it was demonstrated that salidroside was capable of protecting endothelial cells from damage from oxidative stress and mitochondrial dysfunction induced by exogenous ox-LDL. Furthermore, the investigation focused on the underlying cardioprotective molecular mechanisms of salidroside, which were involved in promoting the AMPK/SIRT1 pathway.

SIRT1, a member of the conserved sirtuin family, serves a pivotal role in the pathogenesis and therapy of heart disease through a variety of mechanisms, including reducing apoptosis, improving endothelial function, and defending against oxidative stress $(19,20)$. During the recent years, a number of investigators have proved that salidroside exhibits a cardioprotective effect against atherosclerosis $(12,17,36)$. Notably, evidence confirms that SIRT1 participates in the beneficial effect of salidroside in lung injury and nervous system disease through inhibiting apoptosis, inflammation, and oxidative stress (37-39). However, the role of SIRT1 in the cardioprotective effects of salidroside has not yet been reported. In the present study, it was demonstrated that salidroside mitigates ox-LDL-induced HUVEC injury as demonstrated by the increase of cell viability and downregulation of LDH release. Notably, salidroside reversed the ox-LDL-induced decrease in the expression of the SIRT1 protein, which was in line with the previous study by Donato et al (40), indicating the possible involvement of SIRT1 in the cardioprotective effect of salidroside in atherosclerosis. In addition, the present study confirmed that knockdown of SIRT1 by siSIRT1 transfection inhibits the salidroside-induced cardioprotective effect against ox-LDL-induced HUVECs injury. These results suggested that SIRT1 mediates the cardioprotective effect of salidroside against atherosclerosis.

A number of studies have revealed that oxidative stress is a pivotal feature of atherosclerosis and is produced by an imbalance between antioxidant capability and the presence of species including ROS $(41,42)$. These conditions cause cell injury and serve a critical role in apoptosis via cell death signaling pathways (43). Salidroside can improve cardiac dysfunction by suppressing excessive oxidative stress and cardiomyocyte apoptosis $(33,44)$. However, there are few studies about the effects of salidroside on oxidative stress 
A

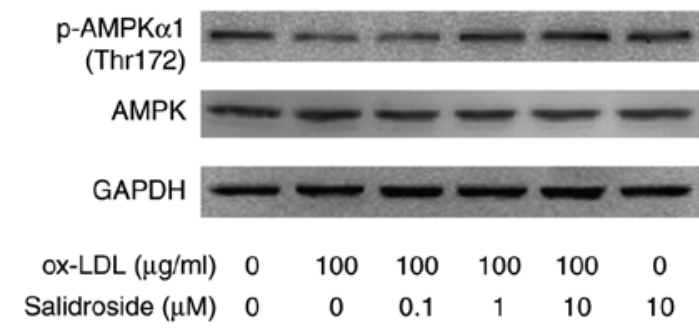

C

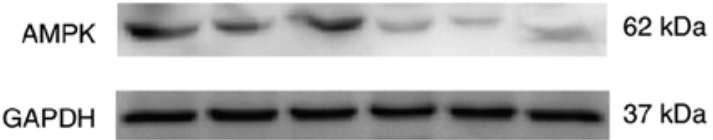

E
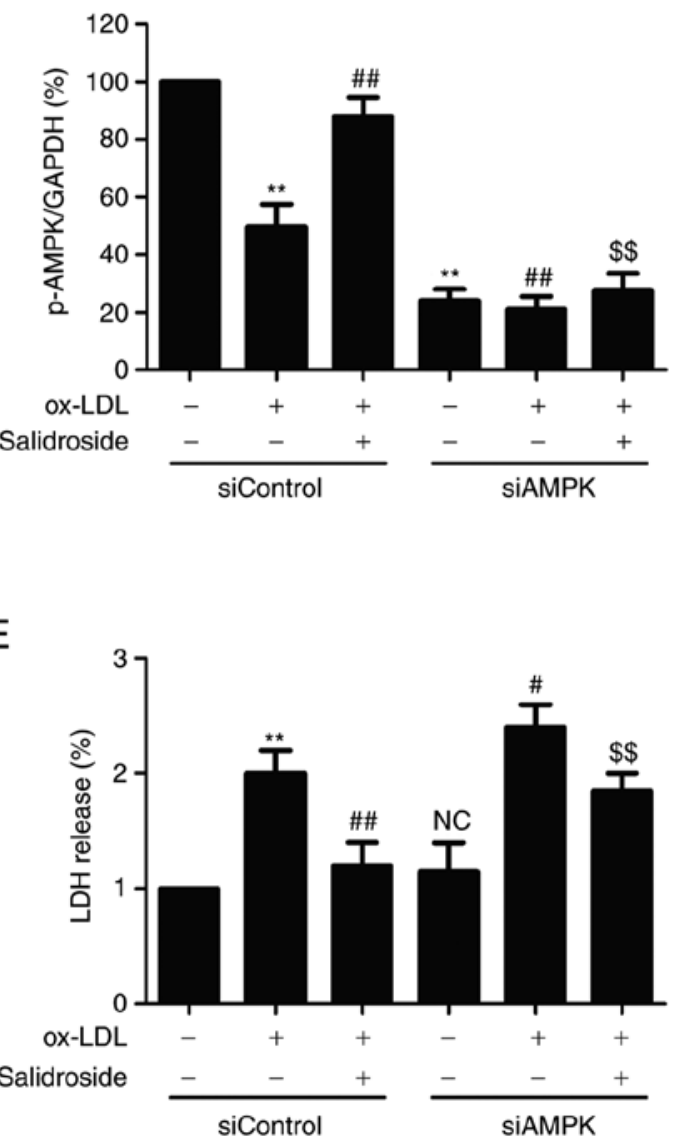

B

$62 \mathrm{kDa}$

$62 \mathrm{kDa}$

$37 \mathrm{kDa}$

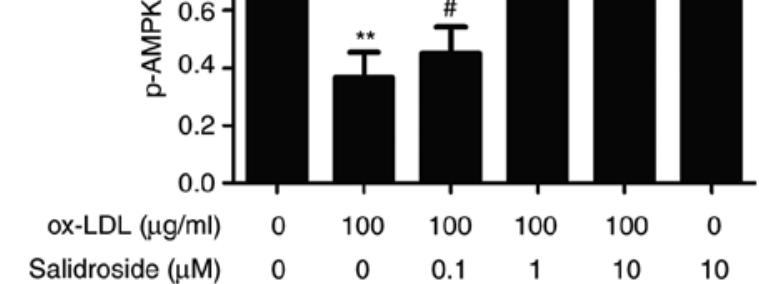

$\mathrm{D}$

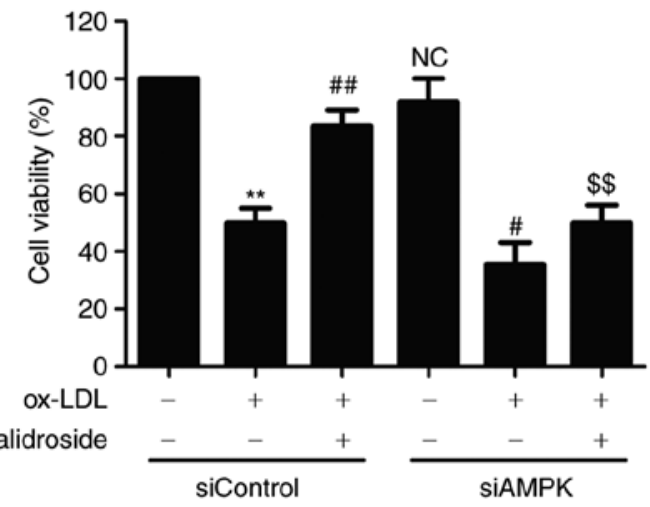

F
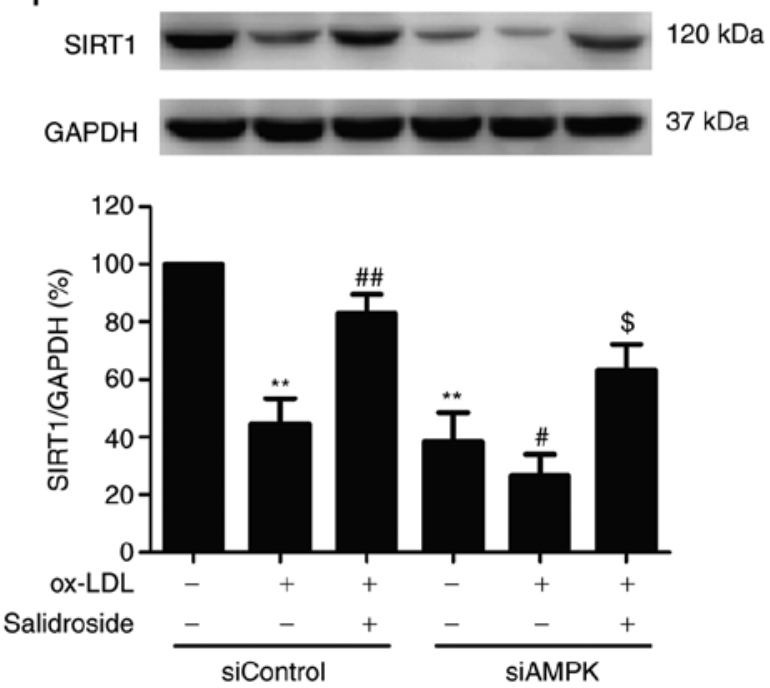

Figure 5. Effects of AMPK knockdown on the protection of salidroside on ox-LDL-induced HUVECs injury and SIRT1 expression in HUVECs. (A) Cells were pretreated with salidroside $(0.1,1$, or $10 \mu \mathrm{M})$ for $1 \mathrm{~h}$ and then stimulated with ox-LDL (100 $\mu \mathrm{g} / \mathrm{ml})$ for $24 \mathrm{~h}$, and the expression of p-AMPK and AMPK were measured by western blot analysis. (B) Bar charts illustrate the quantification of p-AMPK and AMPK proteins. HUVECs were pre-transfected with siAMPK or siControl and incubated with salidroside $(10 \mu \mathrm{M})$ for $1 \mathrm{~h}$ followed by treatment with ox-LDL $(100 \mu \mathrm{g} / \mathrm{ml})$ for $24 \mathrm{~h}$. (C) The transfection efficiency was determined by western blot analysis. (D) The viability was detected using MTT assay. (E) The LDH release was determined using LDH Cytotoxicity Assay kit. (F) The expression of SIRT1 was determined by western blot analysis. Data are expressed as the mean \pm standard; $\mathrm{n}=3$. ${ }^{* *} \mathrm{P}<0.01$ vs. the control group; ${ }^{\#} \mathrm{P}<0.05$ and ${ }^{\# \#} \mathrm{P}<0.01$ vs. the ox-LDL treatment group; ${ }^{\$} \mathrm{P}<0.05$ and ${ }^{\$ \$} \mathrm{P}<0.01$ vs. the ox-LDL and salidroside co-treatment group. p, phosphorylated; AMPK, adenosine monophosphate-activated protein kinase; HUVECs, human umbilical vascular endothelial cells; ox-LDL, oxidized low density lipoprotein; si, small interfering; SIRT1, sirtuin 1; LDH, lactate dehydrogenase; NC, not significant.

and apoptosis in atherosclerosis. In the current study, it was demonstrated that salidroside reduces ox-LDL-induced oxidative stress as demonstrated by the decrease of ROS generation, MDA content and NOX2 expression and improves the antioxidant defense system as demonstrated by the upregulation of SOD and GSH-Px activities. On the other hand, it is confirmed in the literature that SIRT1 deficiency in endothelial cells contributes to increased oxidative stress, inflammation, foam cell formation, apoptosis and autophagy, thereby promoting atherosclerosis $(22,45)$, and multiple myocardial protective drugs-induced beneficial processes were mediated by SIRT1 within the molecular circuitry underlying 
endothelial dysfunction via inhibiting oxidative stress in atherosclerosis (46-48). Importantly, a recent study elucidated that SIRT1 mediates salidroside-elicited protection against apoptosis and oxidative stress in Parkinson's disease (37). In line with these studies, the results of the present study also demonstrated that SIRT1 knockdown mitigates salidroside-induced inhibition of oxidative stress and promotion of the antioxidant defense system in ox-LDL-treated HUVECs, suggesting that SIRT1 mediates the protective effect of salidroside against oxidative stress in atherosclerosis.

The mitochondrion is the main source of chronic ROS production under physiological conditions (6). In addition, enhanced mitochondrial ROS generation and functional disorder are conducive to the development of atherosclerosis $(7,49)$. Previous studies revealed that salidroside can rescue mitochondrial dysfunction caused by various stimuli in HUVECs $(50,51)$. Cai et al $(52)$ proved that salidroside protects the liver against ischemia reperfusion injury by regulating the antioxidant response and mitochondrial permeability transition. In addition, salidroside can prevent $\mathrm{H}_{2} \mathrm{O}_{2}$-induced HUVEC injury through promoting mitochondrial function, thereby reducing the overactivation of oxidative stress-associated downstream signaling pathways (50). Furthermore, salidroside can improve endothelial function and alleviate atherosclerosis depending on mitochondria-associated pathways (15). Additionally, research has confirmed the regulatory effect of SIRT1 on mitochondrial dynamics, which has gained much attention $(53,54)$. Ma et al $(55)$ confirmed that SIRT1 activation alleviates cardiac dysfunction via the restoration of mitochondrial biogenesis and function. In line with these studies, the present study demonstrated that knockdown of SIRT1 attenuates the salidroside-induced inhibition on mitochondrial dysfunction induced by ox-LDL as illustrated by the increase in MMP and the decreases in cytochrome $c$ expression and the $\mathrm{Bax} / \mathrm{Bcl}-2$ ratio, indicating the contribution of SIRT1 to the improvement of salidroside on mitochondrial dysfunction in ox-LDL-treated HUVECs.

AMPK is an important metabolic switch that is involved in the modulation of cellular and whole-body metabolism. Previous evidence indicates that AMPK is essential for the maintenance of cardiovascular health and has a regulatory effect on endothelial function and vascular structure $(56,57)$. For instance, activation of AMPK mitigates the generation of ROS induced by mitochondrial dysfunction, endoplasmic reticulum stress and NADPH oxidase (58). The mechanism of action of a number of therapeutic agents commonly used in cardiovascular and diabetic diseases, including metformin, thiazolidinediones and statins, may involve AMPK (59), although the precise downstream pathways remain poorly defined. Activation of AMPK also has a number of potentially beneficial anti-atherosclerotic effects. Notably, the study from Xing et al (15) revealed that the activation of AMPK-dependent pathway contributes to the salidroside-mediated result of improving endothelial function and alleviating atherosclerosis. Similarly, in the present study it was demonstrated that salidroside reversed the ox-LDL-induced increase in the expression of $\mathrm{p}$-AMPK, indicating the that the activation of AMPK was induced by salidroside. In addition, knockdown of AMPK induced by transfection with siAMPK inhibited the cardioprotective effect of salidroside on ox-LDL-induced
HUVEC injury, which was consistent with the study from Zheng et al (60), which demonstrated that the inhibition of AMPK activity induced by the AMPK inhibitor Compound C and siRNA suppressed the beneficial effects of salidroside in hepatocytes. Notably, it was demonstrated that salidroside increases the expression of p-AMPK but has little effect on the level of AMPK in ox-LDL-treated HUVECs, indicating that salidroside elicited a cardioprotective effect against atherosclerosis via directly increasing p-AMPK expression and activating the AMPK pathway. As SIRT1 exerts an $\mathrm{NAD}^{+}$-dependent deacetylase action, its activity is regulated by the proteins modulating the cellular NAD ${ }^{+}$level. Among them, AMPK is an important protein. It has been confirmed that AMPK phosphorylation regulates SIRT1 activity (61). Importantly, the results of the present study demonstrated that knockdown of AMPK also attenuates the salidroside-induced upregulation of SIRT1 in ox-LDL-treated HUVECs. These results indicated that salidroside improves AMPK activation, which causes SIRT1 expression, leading to cardioprotection in atherosclerosis.

However, there are still certain limitations in the present study, which require further explanation. First, the effects of a wide range of salidroside concentrations $(0.1,1$ and $10 \mu \mathrm{M})$ on cell viability under ox-LDL condition were investigated, but a more accurate concentration of salidroside on the viability of ox-LDL-treated HUVECs is required. In addition, only the effect of siAMPK on SIRT1 expression was demonstrated, whether AMPK affects SIRT1 expression directly or indirectly was not very clear. This will be investigated by co-immunoprecipitation in the authors' future studies. In addition, in the present study, only the effect of salidroside on ox-LDL induced endothelial injures were investigated not the impact of salidroside on endothelial function under ox-LDL conditions. In addition, the results of the present study were based on HUVECs in vitro, whether the AMPK/SIRT1 pathway contributes to the cardioprotective effect of salidroside on atherosclerosis in vivo is needed to be validated further, which is the main focus of future studies. In conclusion, all the findings from the current study indicated that salidroside protects HUVECs from injury caused by ox-LDL by inhibiting oxidation and improving mitochondrial function. The cardioprotection elicited by salidroside is focused on promoting the AMPK/SIRT1 pathway. The results of the present study will provide experimental evidence for the future clinical applications of salidroside to prevent atherosclerosis, giving new insights into the cardiovascular benefits of the AMPK/SIRT1 pathway.

\section{Acknowledgements}

Not applicable.

\section{Funding}

No funding received.

\section{Availability of data and materials}

All data generated or analyzed during the present study are included in this published article. 


\section{Authors' contributions}

DZ performed the experiment and was the major contributor in writing the manuscript. XS, SL and MS analyzed and interpreted the data. HG, YZ and ZW contributed to the design the work and to article revision. PD, LZ and MY made substantial contributions to the conception and design of the study. XW designed the entire experiment, revised the article for publication and gave final approval of the version to be published. All authors read and approved the final manuscript.

\section{Ethics approval and consent to participate}

Not applicable.

\section{Patient consent for publication}

Not applicable.

\section{Competing interests}

The authors declare that they have no competing interests.

\section{References}

1. Libby P, Ridker PM and Hansson GK: Progress and challenges in translating the biology of atherosclerosis. Nature 473: 317-325, 2011.

2. Gao S and Liu J: Association between circulating oxidized low-density lipoprotein and atherosclerotic cardiovascular disease. Chronic Dis Transl Med 3: 89-94, 2017.

3. Pirillo A, Norata GD and Catapano AL: LOX-1, OxLDL, and atherosclerosis. Mediators Inflamm 2013: 152786, 2013.

4. Pahwa R and Jialal I: Atherosclerosis. StatPearls Publishing Treasure Island, FL, 2019.

5. Taniyama Y and Griendling KK: Reactive oxygen species in the vasculature: Molecular and cellular mechanisms. Hypertension 42: 1075-1081, 2003.

6. Sorescu D and Griendling KK: Reactive oxygen species, mitochondria, and $\mathrm{NAD}(\mathrm{P}) \mathrm{H}$ oxidases in the development and progression of heart failure. Congest Heart Fail 8: 132-140, 2002.

7. Madamanchi NR and Runge MS: Mitochondrial dysfunction in atherosclerosis. Circ Res 100: 460-473, 2007.

8. Panth N, Paudel KR and Parajuli K: Reactive oxygen species: A key hallmark of cardiovascular disease. Adv Med 2016: 9152732 , 2016.

9. Victor VM, Apostolova N, Herance R, Hernandez-Mijares A and Rocha M: Oxidative stress and mitochondrial dysfunction in atherosclerosis: Mitochondria-targeted antioxidants as potential therapy. Curr Med Chem 16: 4654-4667, 2009.

10. Lum H and Roebuck KA: Oxidant stress and endothelial cell dysfunction. Am J Physiol Cell Physiol 280: C719-C741, 2001.

11. Sun P, Song SZ, Jiang S, Li X, Yao YL, Wu YL, Lian LH and Nan JX: Salidroside regulates inflammatory response in raw 264.7 macrophages via TLR4/TAK1 and ameliorates inflammation in alcohol binge drinking-induced liver injury. Molecules 21: E1490, 2016.

12. Ni J, Li Y, Li W and Guo R: Salidroside protects against foam cell formation and apoptosis, possibly via the MAPK and AKT signaling pathways. Lipids Health Dis 16: 198, 2017.

13. Ju L, Wen X, Wang C, Wei Y, Peng Y, Ding Y, Feng L and Shu L: Salidroside, a natural antioxidant, improves $\beta$-cell survival and function via activating AMPK pathway. Frontiers Pharmacol 8 : 749, 2017.

14. Zhang P, Li Y, Guo R and Zang W: Salidroside protects against advanced glycation end products-induced vascular endothelial dysfunction. Med Sci Monit 24: 2420-2428, 2018.

15. Xing SS, Yang XY, Zheng T, Li WJ, Wu D, Chi JY, Bian F, Bai XL, Wu GJ, Zhang YZ, et al: Salidroside improves endothelial function and alleviates atherosclerosis by activating a mitochondria-related AMPK/PI3K/Akt/eNOS pathway. Vascul Pharmacol 72: 141-152, 2015.
16. Panossian A, Hamm R, Wikman G and Efferth T: Mechanism of action of Rhodiola, salidroside, tyrosol and triandrin in isolated neuroglial cells: An interactive pathway analysis of the downstream effects using RNA microarray data. Phytomedicine 21: 1325-1348, 2014.

17. Xing SS, Li J, Chen L, Yang YF, He PL,Li J and Yang J: Salidroside attenuates endothelial cellular senescence via decreasing the expression of inflammatory cytokines and increasing the expression of SIRT3. Mech Ageing Dev 175: 1-6, 2018.

18. Kitada M, Ogura Y and Koya D: The protective role of Sirt1 in vascular tissue: Its relationship to vascular aging and atherosclerosis. Aging 8: 2290-2307, 2016.

19. Winnik S, Auwerx J, Sinclair DA and Matter CM: Protective effects of sirtuins in cardiovascular diseases: From bench to bedside. Eur Heart J 36: 3404-3412, 2015.

20. Ma L and Li Y: SIRT1: Role in cardiovascular biology. Clin Chim Acta 440: 8-15, 2015.

21. Chong ZZ, Shang YC, Wang S and Maiese K: SIRT1: New avenues of discovery for disorders of oxidative stress. Expert Opin Ther Targets 16: 167-178, 2012.

22. Ota H, Eto M, Ogawa S, Iijima K, Akishita M and Ouchi Y: SIRT1/eNOS axis as a potential target against vascular senescence, dysfunction and atherosclerosis. J Atheroscler Thromb 17: 431-435, 2010.

23. Yang L, Cong HL, Wang SF and Liu T: AMP-activated protein kinase mediates the effects of lipoprotein-associated phospholipase A2 on endothelial dysfunction in atherosclerosis. Exp Ther Med 13: 1622-1629, 2017.

24. Wang S, Song P and Zou MH: AMP-activated protein kinase, stress responses and cardiovascular diseases. Clin Sci 122: 555-573, 2012.

25. Canto C, Jiang LQ, Deshmukh AS, Mataki C, Coste A, Lagouge M, Zierath JR and Auwerx J: Interdependence of AMPK and SIRT1 for metabolic adaptation to fasting and exercise in skeletal muscle. Cell Metab 11: 213-219, 2010.

26. Stein S and Matter CM: Protective roles of SIRT1 in atherosclerosis. Cell Cycle 10: 640-647, 2011.

27. Martin-Ventura JL, Rodrigues-Diez R, Martinez-Lopez D, Salaices M, Blanco-Colio LM and Briones AM: Oxidative stress in human atherothrombosis: Sources, markers and therapeutic targets. Int J Mol Sci 18: E2315, 2017.

28. Georgieva E, Ivanova D, Zhelev Z, Bakalova R, Gulubova M and Aoki I: Mitochondrial dysfunction and redox imbalance as a diagnostic marker of 'Free Radical Diseases'. Anticancer Res 37: 5373-5381, 2017

29. Vásquez-Trincado C, García-Carvajal I, Pennanen C, Parra V, Hill JA, Rothermel BA and Lavandero S: Mitochondrial dynamics, mitophagy and cardiovascular disease. J Physiol 594: 509-525, 2016

30. Salminen A, Kaarniranta K and Kauppinen A: Age-related changes in AMPK activation: Role for AMPK phosphatases and inhibitory phosphorylation by upstream signaling pathways. Ageing Res Rev 28: 15-26, 2016.

31. Mudau M, Genis A, Lochner A and Strijdom H: Endothelial dysfunction: The early predictor of atherosclerosis. Cardiovasc J Afr 23: 222-231, 2012.

32. Zou H, Liu X, Han T, Hu D, Wang Y, Yuan Y, Gu J, Bian J, Zhu J and Liu ZP: Salidroside protects against cadmium-induced hepatotoxicity in rats via GJIC and MAPK pathways. PLoS One 10: $\mathrm{e} 0129788,2015$.

33. Zhu Y, Shi YP, Wu D, Ji YJ, Wang X, Chen HL, Wu SS, Huang DJ and Jiang W: Salidroside protects against hydrogen peroxide-induced injury in cardiac $\mathrm{H} 9 \mathrm{c} 2$ cells via PI3K-Akt dependent pathway. DNA Cell Biol 30: 809-819, 2011.

34. Tan CB, Gao M, Xu WR, Yang XY, Zhu XM and Du GH: Protective effects of salidroside on endothelial cell apoptosis induced by cobalt chloride. Biol Pharm Bull 32: 1359-1363, 2009.

35. Wu YL, Piao DM, Han XH and Nan JX: Protective effects of salidroside against acetaminophen-induced toxicity in mice. Biol Pharm Bull 31: 1523-1529, 2008.

36. Sun L, Dou F, Chen J, Chi H, Xing S, Liu T, Sun S and Chen C: Salidroside slows the progression of EA.hy926 cell senescence by regulating the cell cycle in an atherosclerosis model. Mol Med Rep 17: 257-263, 2018.

37. Wang CY, Sun ZN, Wang MX and Zhang C: SIRT1 mediates salidroside-elicited protective effects against $\mathrm{MPP}^{+}$-induced apoptosis and oxidative stress in SH-SY5Y cells: Involvement in suppressing MAPK pathways. Cell Biol Int 42: 84-94, 2018. 
38. Wang Y, Xu CF, Liu YJ, Mao YF, Lv Z, Li SY, Zhu XY and Jiang L: Salidroside attenuates ventilation induced lung injury via SIRT1-dependent inhibition of NLRP3 inflammasome. Cell Physiol Biochem 42: 34-43, 2017.

39. Si PP, Zhen JL, Cai YL, Wang WJ and Wang WP: Salidroside protects against kainic acid-induced status epilepticus via suppressing oxidative stress. Neurosci Lett 618: 19-24, 2016.

40. Donato AJ, Magerko KA, Lawson BR, Durrant JR, Lesniewski LA and Seals DR: SIRT-1 and vascular endothelial dysfunction with ageing in mice and humans. J Physiol 589: 4545-4554, 2011.

41. Yang X, Li Y, Li Y, Ren X, Zhang X, Hu D, Gao Y, Xing Y and Shang H: Oxidative stress-mediated atherosclerosis: Mechanisms and therapies. Front Physiol 8: 600, 2017.

42. Zhang M, Pan H, Xu Y, Wang X, Qiu Z and Jiang L: Allicin decreases lipopolysaccharide-induced oxidative stress and inflammation in human umbilical vein endothelial cells through suppression of mitochondrial dysfunction and activation of $\mathrm{Nrf} 2$. Cell Physiol Biochem 41: 2255-2267, 2017.

43. Mukherjee N, Parida PK, Santra A, Ghosh T, Dutta A, Jana K, Misra AK and Sinha Babu SP: Oxidative stress plays major role in mediating apoptosis in filarial nematode Setaria cervi in the presence of trans-stilbene derivatives. Free Radic Biol Med 93: 130-144, 2016.

44. Wang XL, Wang X, Xiong LL, Zhu Y, Chen HL, Chen JX, Wang XX, Li RL, Guo ZY, Li P, et al: Salidroside improves doxorubicin-induced cardiac dysfunction by suppression of excessive oxidative stress and cardiomyocyte apoptosis. J Cardiovasc Pharmacol 62: 512-523, 2013.

45. Sosnowska B, Mazidi M, Penson P, Gluba-Brzózka A, Rysz J and Banach M: The sirtuin family members SIRT1, SIRT3 and SIRT6: Their role in vascular biology and atherogenesis. Atherosclerosis 265: 275-282, 2017.

46. Tsai KL, Hung CH, Chan SH, Hsieh PL, Ou HC, Cheng YH and Chu PM: Chlorogenic acid protects against oxLDL-induced oxidative damage and mitochondrial dysfunction by modulating SIRT1 in endothelial cells. Mol Nutr Food Res 62: e1700928, 2018.

47. Chan SH, Hung CH, Shih JY, Chu PM, Cheng YH, Lin HC, Hsieh PL and Tsai KL: Exercise intervention attenuates hyperhomocysteinemia-induced aortic endothelial oxidative injury by regulating SIRT1 through mitigating NADPH oxidase/LOX-1 signaling. Redox Biol 14: 116-125, 2018.

48. Zhu X, Yue H, Guo X, Yang J, Liu J, Liu J, Wang R and Zhu W: The preconditioning of berberine suppresses hydrogen peroxide-induced premature senescence via regulation of Sirtuin 1. Oxid Med Cell Longev 2017: 2391820, 2017.

49. Ballinger SW, Patterson C, Knight-Lozano CA, Burow DL, Conklin CA, Hu Z, Reuf J, Horaist C, Lebovitz R, Hunter GC, et al: Mitochondrial integrity and function in atherogenesis. Circulation 106: 544-549, 2002.
50. Xing S, Yang X, Li W, Bian F, Wu D, Chi J, Xu G, Zhang Y and Jin S: Salidroside stimulates mitochondrial biogenesis and protects against $\mathrm{H}_{2} \mathrm{O}_{2}$-induced endothelial dysfunction. Oxid Med Cell Longev 2014: 904834, 2014.

51. Xu MC, Shi HM, Wang H and Gao XF: Salidroside protects against hydrogen peroxide-induced injury in HUVECs via the regulation of REDD1 and mTOR activation. Mol Med Rep 8: 147-153, 2013.

52. Cai L, Li Y, Zhang Q, Sun H, Yan X, Hua T, Zhu Q, Xu H and Fu H: Salidroside protects rat liver against ischemia/reperfusion injury by regulating the GSK-3 $3 / \mathrm{Nrf} 2$-dependent antioxidant response and mitochondrial permeability transition. Eu J Pharmacol 806: 32-42, 2017.

53. Dolinsky VW: The role of sirtuins in mitochondrial function and doxorubicin-induced cardiac dysfunction. Biol Chem 398: 955-974, 2017.

54. Tang BL: Sirtl and the Mitochondria. Mol Cells 39: 87-95, 2016

55. Ma S, Feng J, Zhang R, Chen J, Han D, Li X, Yang B, Li X, Fan M, Li C, et al: SIRT1 activation by resveratrol alleviates cardiac dysfunction via mitochondrial regulation in diabetic cardiomyopathy mice. Oxid Med Cell Longev 2017: 4602715, 2017.

56. Bairwa SC, Parajuli N and Dyck JR: The role of AMPK in cardiomyocyte health and survival. Biochim Biophys Acta 1862: 2199-2210, 2016

57. Shirwany NA and Zou MH: AMPK in cardiovascular health and disease. Acta Pharmacol Sin 31: 1075-1084, 2010.

58. Gao F, Chen J and Zhu H: A potential strategy for treating atherosclerosis: Improving endothelial function via AMP-activated protein kinase. Sci China Life Sci 61: 1024-1029, 2018.

59. Ewart MA and Kennedy S: AMPK and vasculoprotection. Pharmacol Ther 131: 242-253, 2011.

60. Zheng T, Yang X, Li W, Wang Q, Chen L, Wu D, Bian F, Xing S and Jin S: Salidroside attenuates high-fat diet-induced nonalcoholic fatty liver disease via AMPK-dependent TXNIP/NLRP3 pathway. Oxid Med Cell Longev 2018: 8597897, 2018.

61. Ruderman NB, Xu XJ, Nelson L, Cacicedo JM, Saha AK, Lan F and Ido Y: AMPK and SIRT1: A long-standing partnership? Am J Physiol Endocrinol Metab 298: E751-E760, 2010.

This work is licensed under a Creative Commons Attribution-NonCommercial-NoDerivatives 4.0 International (CC BY-NC-ND 4.0) License. 